\title{
The principle of QALY maximisation as the basis for allocating health care resources
}

\author{
John Cubbon Birmingham University
}

\section{Author's abstract}

This paper presents a case for allocating health care resources so as to maximise Quality Adjusted Life Years (QALYs). Throughout parallels are drawn with the grounds for adopting utilitarianism. QALYs are desirable because they are essential for human flourishing and goal-attainment. In conditions of scarcity the principle of QALY maximisation may involve unequal treatment of different groups of people; and it is argued that this is not objectionable. Doctors in their dealings with patients should not be continually consulting the principle (though it can sometimes be useful); instead by following existing ethical codes more $Q A L Y$ s will be produced overall. In the formulation of policy, however, the principle should be applied in a thoroughgoing way and, if it is, it will not have some of the counterintuitive consequences it may have in interpersonal situations.

\section{The principle of QALY maximisation}

The QALY has become well known in the health service largely as a result of work at the Centre for Health Economics at the University of York. Survival data have been used for some time in measuring the benefits of treatment; QALYs by contrast depend not only on the number of years gained but also on their quality. Rosser's Classification of Illness States (1) is frequently used to construct them. It has in effect two dimensions - observed disability (loss of function and mobility) and subjective distress - which constitute a reasonable operationalisation of degrees of severity of ill-health, though they do not provide a comprehensive measure of the quality of life, nor are they intended to. Quality of life has many other components: enjoyment derived from one's customary activities, relationships with others, protection from the elements, and so on. It would have to be measured, if indeed it can be at all, by a hotch-potch of disparate scales, which would vary from culture to culture, from sub-culture to subculture and even from individual to individual. Although QALYs do not quantify the quality of life in

\section{Key words}

QALYs; quality of life; policy-making. its all-embracing sense, they measure those of its dimensions which are of primary importance in health. care. It is possible to use QALYs as the building-blocks of health policy in a number of ways (2); but generally $\infty$ the approach adopted is to implement the course of 0 action which results in more QALYs than any alternative. This is the principle of QALY maximisation. A fairly clear example of its application is provided by Williams's (3) seminal article on coronary artery bypass grafting. He advocates the marginal redeployment of resources to procedures for which the benefit in terms of QALYs is high in relation to costs. By this criterion hip replacement and the insertion of pacemakers for heart-block take priorit over the provision of additional facilities for patients i⿱⿱一𫝀口亍 need of kidney transplants.

\section{The basis for adopting the principle}

The principle of QALY maximisation may follow from utilitarianism or a need-based moral theory. There are, in fact, several routes of argument to it; and its supporters have not necessarily inferred it, as Harris (4) suggests, from a simple preference shared by most people. Harris imagines two individuals who would rather have a year of healthy life than three years of $:$ severe discomfort. Only one of them can be saved from immediate death. Being saved will mean three years of $\dot{\partial}$ discomfort for one; and for the other a year of health. Harris points out that it does not follow that both are 0 bound in some way to infer that the individual who would have the shorter, healthier life should be saved. This, incidentally, is reminiscent of a standard $\underset{N}{N}$ purported refutation of Mill's 'proof of the principle of $O$ utility' in chapter IV of Utilitarianism $(5,6)$. The $N$ argument might be put in more general terms as the $\underset{\mathrm{N}}{N}$ denial of the following inference:

1. Each person in a given population desires as many QALYs as possible for himself or herself.

2 . Therefore each person is morally bound to choose 7 the option which will produce the maximum number of QALYs in the population even if it gives him or her, $\mathbb{D}$ as an individual, fewer QALYs than some alternative.

Clearly 1. does not imply 2. but the adherent of QALY maximisation does not need to rest his case on 1 . 
Comparison with Mill's proof may be illuminating here. If each person's happiness is a good for him or her it does not follow that the happiness of everyone is a good for each person. Clearly if Mill had drawn this inference, he would have committed a fallacy. His argument can be interpreted or reconstructed as a move from the premise that giving someone what he or she wants is good to the conclusion that the maximum good will be produced by satisfying as many desires as possible. Likewise the defender of QALY maximisation can claim that the principle rests on the judgement that raising someone's stock of QALYs is a good for him or her.

The next question to answer is: What are the grounds for such a judgement? It is at this point that one finds oneself knocking against one's most fundamental moral intuitions and there will doubtless be many who will never be persuaded that QALYs are significant; however, reflection on the fact that they are a basic human need in all times and places may sway some of the waverers. The liberal freedoms of speech, thought and action have also been claimed to be necessary for the attainment of the goals of rational beings; and the claim has a considerable degree of force. However, even they may be represented as the products of a particular value-system. It would, however, be absurd to suggest that QALYs only matter to people living in Western liberal democracies, advanced capitalist societies, Welfare States or whatever. Throughout history pain and disability have prevented human beings from achieving their aims. If what is essential for human flourishing or the achievement of a person's objectives is to be promoted, the need for QALYs will not be the only one that should be satisfied. Food, companionship, fulfilling activities and so forth are equally basic needs, but in the formulation of health care policies, they are presumed to be either satisfied or of lesser importance or randomised. In other words, when it is a question of deciding on actions affecting sick people, their primary need is to be made healthy.

The appeal of QALYs can be brought out by a contrast with an alternative position, namely that what is valuable are lives, not life-years or their quality. From this point of view society should aim purely and simply to keep the number of deaths to a minimum. It would follow that one should strive to save a baby who can only live another hour of acute suffering just as much as one who will have a happy and fruitful existence for three score years and ten. The two individuals are both human and subjects of consciousness; and surely at least some of the significance that is attributed to these characteristics is adventitious. An evaluation of life or lives without regard to actual or potential QALYs seems very incomplete. QALY maximisation on the other hand views QALYs as an essential prerequisite for what we regard as important in our lives. Surely health is a sine qua non for the whole gamut of activities, experiences, aspirations and attainment of goals which make our lives valuable to us.

\section{Unequal treatment of groups of patients}

It may be objected that the principle discriminates against those less capable of deriving benefit from health care resources. Harris (4) propounds this objection and argues that everyone has an equal right to be kept alive. As Williams (7) so rightly implies, the difference between the two viewpoints is a fundamental ethical one and cannot be conclusively settled by argument. What can be done is to adduce considerations in support of one or other of them. It can only be contingently true that particular categories of people will tend to be disadvantaged by the principle (8). It is not primarily because of their heart condition that patients needing a heart transplant would not be given the highest priority in the allocation of resources, but because the money spent on treating them would generate more QALYs if it were directed to another category of sick people. Moreover discrimination loses some of its sting if those who are less favourably treated are not members of a clearly defined group with a corporate sense. Those with conditions for which treatment costs more on average for each QALY gained constitute a collectivity which is both highly disparate and, because of medical advances, continually changing. People needing coronary artery bypass grafting for mild angina with double-vessel disease - which is expensive in QALY terms - feel much less affinity for one another than members of ethnic minorities.

Triage in time of war can be represented as a pointed illustration of the implementation of the principle. In general, priority is given to those needing a quick, straightforward life-saving operation and anyone with multiple wounds for whom little can be done may, in consequence, be left to die. This policy may often be motivated by the need to return as many men as possible to the front-line, but it will produce incomparably more QALYs than the selection of patients on a more equitable basis - for example, by drawing lots, as Harris (9) suggests. A choice has to be made between a type of fairness and QALY maximisation. In war the scarce resource is time; in the health service in Britain today it is diffuse and multifaceted and, as a result, the inefficiency of its distribution from the point of view of generating QALYs is less apparent. War raises the issue of resource allocation more starkly than usual; but is there a good reason why the criterion for deciding on priorities should really be different in peacetime?

\section{The context within which the principle should be applied}

If the context within which QALY maximisation has a role to play is properly delineated, some of the objections that have been raised against it can be seen to be misdirected. It is primarily to be used in policy decisions about resource allocation and it should be far more sparingly applied in dealing with individual 
patients. There is no contradiction in this two-level approach; but it needs explanation.

The impersonal calculating aspect of utilitarianism which makes it seem so inappropriate as a morality of personal relations is less objectionable in the formulation of policy for at least two reasons:

1) In a modern society the planning of services will always mean that some groups of people will deliberately be deprived of benefits.

2) Those affected by policies will not generally be known as individuals to those formulating them.

It will be argued that QALY maximisation parallels utilitarianism in these respects.

Technological progress has greatly increased human interdependence and at the same time our lives have come under corporate control to an ever-increasing extent. If disruptions such as wars are set on one side, the consequences of different social policies not only affect large numbers of people, but they are also increasingly predictable. A decision-maker may be aware of a number of options which affect people's QALYs in different ways. Almost always there will be no option which leaves everyone under consideration better-off than each of the alternatives. Because resources are not unlimited, the decision-maker may, for example, need to choose between the introduction of an acute hospital facility and the expansion of a health promotion programme. Because of the complexity of the alternatives he or she will need to make careful calculations of the cost and benefits. He or she will have to choose who will receive the most QALYs and may even have to decide who will live and who will die. The explosion of information on service provision and its impact on the population often means that, like it or not, someone charged with decisions cannot but know the consequences of different actions in terms of approximate costs and benefits. Such a person cannot but 'play God'. In the health service, particularly as resources have been declining, it is becoming ever more apparent that development means using funds for one care-group rather than another with competing claims. Consciously withholding benefits in QALY-terms from particular categories of people is becoming more and more an unavoidable part of health service decision-making. The informed formulation of policies affecting the distribution of scarce resources, though at times painful for those who undertake it, does not go against the grain of our moral intuitions. The people who would benefit from the developments under consideration will mostly not be known to policy-makers. Often they will not be known to anyone, because they have not yet become ill. According to criteria similar to those which defined the Rawlsian Original Position, a judgement as to which policy will maximise QALYs will, therefore, have a measure of objectivity (10). Such a judgement takes place on a different plane from ordinary interpersonal relationships and so should not interfere with the norms of behaviour governing contact with known others; and it is at this micro-level that the universal application of the principle is intuitively much less acceptable; and current practice is sharply at variance with it. This can be seen from a consideration of medical ethics.

Some clinicians are bitterly opposed to QALYs and their opposition is understandable. The overriding principle governing the actions of a doctor towards a patient is that the best should be done for that patient (11). Explicit consideration of what will maximise QALYs will rarely be carried out. The same applies to selection from a waiting-list. In general, priority is given according to the perceived degree of suffering with those at risk of loss of life being treated first (12). Clearly doctors in their dealings with patients are not always taking decisions about the allocation of their time and efforts on the basis of calculations of QALYs produced by various alternative actions. The clinical ethic can operate satisfactorily to a large extent without QALYs, but this is not to say that doctors never consider them. Sometimes there may be several options affecting a patient's quality and quantity of life in different ways - for example, in the treatment of chronic renal failure. In such situations QALYs may be useful.

If QALY maximisation were what all doctors always consciously strove for, it would not be attained. This is because the doctor-patient relationship is, and should be, an individual and personal one. It does not rest on mathematical calculations. Many doctors in the health service are seldom presented with situations in which they have to decide which of their patients to give time, effort and resources to, when this will clearly be to the disadvantage of the others. When they have to take these decisions they may feel they are 'playing God' with people who are known to them, thereby undermining their capacity to relate to them as patients. Smith's (2) valid point that judgements in a clinical context have a personal dimension which cannot be captured in QALY terms may, therefore, be seen not to threaten the principle of QALY maximisation in the sphere in which it is properly to be applied. Paradoxically it is because doctors' behaviour towards their patients tends not to be based on continually totting up QALYs that more QALYs are produced overall. The unsuitability of the goal of QALY maximisation as the sole determinant of doctors' actions is not unlike a well known limitation of the role of the principle of utility. If, instead of seeping up the motley collection of rules-of-thumb, Biblical commandments, taboos and so on which together constitute popular morality, everyone were taught to act on the imperative 'maximise utility', and only on that imperative, in deciding how to act, less utility would be produced. Similarly the bundles of rules followed by doctors in clinical situations, though some may led to actions that do not in every case maximise QALYs, produce more QALYs overall than would be the case if they were universally replaced by the 
principle that everything should always be done to maximise QALYs.

At the individual level, there are competing considerations - individual preferences, idiosyncratic lifestyles and what Glover (13) calls 'side-effects'. In policy formulation these factors can usually be treated as random and so not taken account of. In decisions of policy in the sphere of health care the main predictable and achievable results are an increase in health or a reduction in illness; and, more importantly, the quality of life of those affected by the policies on offer will in general be enhanced by an improvement in their health more than anything else. At the same time, the QALY does not purport to be all-embracing. For example, an imprisoned ailing scientist may be better off with access to a laboratory than with a higher level of mobility. However, when someone is ill or potentially ill, his or her quality of life will, ceteris paribus, be raised by an improvement in health more than any other change subject to human control. Therefore if one summed the gains in life-years adjusted for all aspects of the quality of life which would result from the implementation of QALY maximisation, as a policy aimed at sick people, the total would be greater than it would be under any alternative (even though the number of sick people who benefited might not be). Griffin's physically handicapped person who prefers an education in philosophy to lifts and wheelchairs may be better off having his preference satisfied (14). Such people are, however, a minority. Consequently more of an increase in the quality of life will be brought about if the state provides, as a matter of policy, aids and appliances, rather than philosophical training, for disabled people. In choices between individuals, the side-effects of saving one person rather than another in terms of the impact on dependants and contribution to society are difficult to write off as irrelevant; and yet it may seem invidious and even dangerous to establish a pecking-order of value on the basis of such considerations (13). This thorny issue can largely be set aside in policy formulation, since for the most part it may be assumed that types of people with different capacities for benefiting and harming others would be equally likely to need different types of treatment.

\section{Conclusion}

As well as attempting to bring out the appeal of the QALY as a building-block in health care ethics, this article is intended to delineate the sphere within which the principle of QALY maximisation should be employed. My argument has been that the principle ought only to be sparingly applied in clinical decisionmaking. It is hoped that the recognition that QALYs have a more restricted role than has sometimes been assumed may reduce some of the hostility towards them.

\section{Acknowledgements}

The author would like to thank Professor E G Knox, Miss K Walker and Professor R K Griffiths for their comments on an earlier version of this paper and Dr B $\mathrm{J}$ Crump for a useful discussion.

f E Cubbon, MA, BPhil, MSc, is Research and $\vec{\forall}$ Information Officer, Department of Public Health $\rightarrow$ Medicine, Central Birmingham Health Authority and $\vec{\infty}$ Honorary Research Fellow, University of Birmingham.

\section{References}

(1) Rosser R M, Watts V C. The measurement of hospital output. International journal of epidemiology 1972; 1: 361-366.

(2) Smith A. Qualms about QALYs. Lancet 1987; i: 1134 1136.

(3) Williams A. Economics of coronary artery bypass grafting. British medical journal 1985; 291: 326-329. 은

(4) Harris J. QALY fying the value of life. Fournal of medic ethics 1987; 13: 117-123.

(5) Mill J S. Utilitarianism. London: Dent, 1961.

(6) Sidgwick H. Methods of ethics (7th ed). London: Macmillan and Co, 1930.

(7) Williams A. Response: QALYfying the value of life. Journal of medical ethics 1987; 13: 123.

(8) Williams A. Quality-Adjusted Life-Years. Lancet 1987; ii: 1372 .

(9) Harris J. EQALYty. In: Byrne P, ed. Health, rights and resources: King's College studies 1987-8. London: King Edward's Hospital Fund for London, 1988: 100-127.

(10) Rawls J. A theory of justice. Cambridge, Mass: Harvard University Press, 1971.

(11) Devlin B. Economists and clinicians: the crucial debate. Health and social service journal 1985 Jul 18: (Centre Eight).

(12) Rawles J. Castigating QALYs. Fournal of medical ethics 1989; 15: 143-147. (13) Glover J. Causing death and saving lives.

(14) Griffin J. Well-being: its meaning, measurement and moral ô importance. Oxford: Clarendon Press, 1986. 